\title{
Self-Management and Transition Among Adolescents/Young Adults with Chronic or End-Stage Kidney Disease
}

\author{
Maria E. Ferris ${ }^{a}$ Jessica R. Cuttance ${ }^{b}$ Karina Javalkar ${ }^{c}$ Sarah E. Cohen $^{b}$ \\ Alexandra Phillips $^{\text {a }}$ Kristi Bickford $^{a}$ Keisha Gibson ${ }^{a} \quad$ M. Ted Ferris ${ }^{a}$ \\ Karin True ${ }^{\mathrm{a}}$ \\ a University of North Carolina Kidney Center at Chapel Hill, ${ }^{b}$ University of North Carolina School of Allied Health, and \\ 'University of North Carolina School of Public Health, Chapel Hill, N.C., USA
}

\section{Key Words}

Transition · Health-care transition · Self-management ·

Adolescents · Young adults · CKD · ESKD - Transition

readiness $\cdot$ Illness self-management

\begin{abstract}
Background: Adolescents/young adults (AYA) with chronic kidney disease (CKD) or end-stage kidney disease (ESKD) are at risk for poor health outcomes related to self-management. To improve their health and quality of life, AYA must build self-management (for those in the pediatric-and adultfocused setting) and/or health care transition (HCT) skills (for those in the pediatric setting). Methods: Self-management and/or HCT encompass a variety of domains that must be tailored to each individual. Annual assessments should begin between the ages of 12 and 14 and continue in the adultfocused setting until patients have achieved demonstrated self-management and/or HCT skills mastery. These assessments will guide interventions that are congruent in terms of literacy, development and culture. Facilitation of this process from the perspective of both the pediatric and the adult health-care systems is described. Conclusions: Deficiencies
\end{abstract}

(C) 2015 S. Karger AG Basel

0253-5068/15/0393-0099\$39.50/0 and barriers to self-management and/or HCT for AYA with ESKD remain. There is no consensus on the definition of successful HCT preparation, with few tools to assess transition readiness and/or self-management. It is important for health providers to promote the self-management and/or healthcare transition skills of AYA with ESKD. Customization of these activities and involvement of the whole family will contribute towards better health-related quality of life and patient outcomes.

(c) 2015 S. Karger AG, Basel

\section{Introduction}

Adolescents/young adults (AYA) constitute approximately $3 \%$ of the end-stage kidney disease (ESKD) population. These patients have a superior 10-year survival compared with older adults [1], but are at a greater risk for poor health self-management and transplant outcomes $[2,3]$. AYA with renal disease receiving health services in the pediatric- or adult-focused settings must learn how to manage their condition to avoid complications or preventable comorbidities. The purpose of this manu-

\section{KARGER 125}

E-Mail karger@karger.com www.karger.com/bpu
Maria E. Ferris, MD, MPH, $\mathrm{PhD}$

University of North Carolina at Chapel Hill

7021 Burnett Womack

Chapel Hill, NC 27599-7155 (USA)

E-Mail Maria_ferris@med.unc.edu 
Fig. 1. Number of patients with end-stage kidney disease between 10-29 years of age reported in the United States Renal Data System.

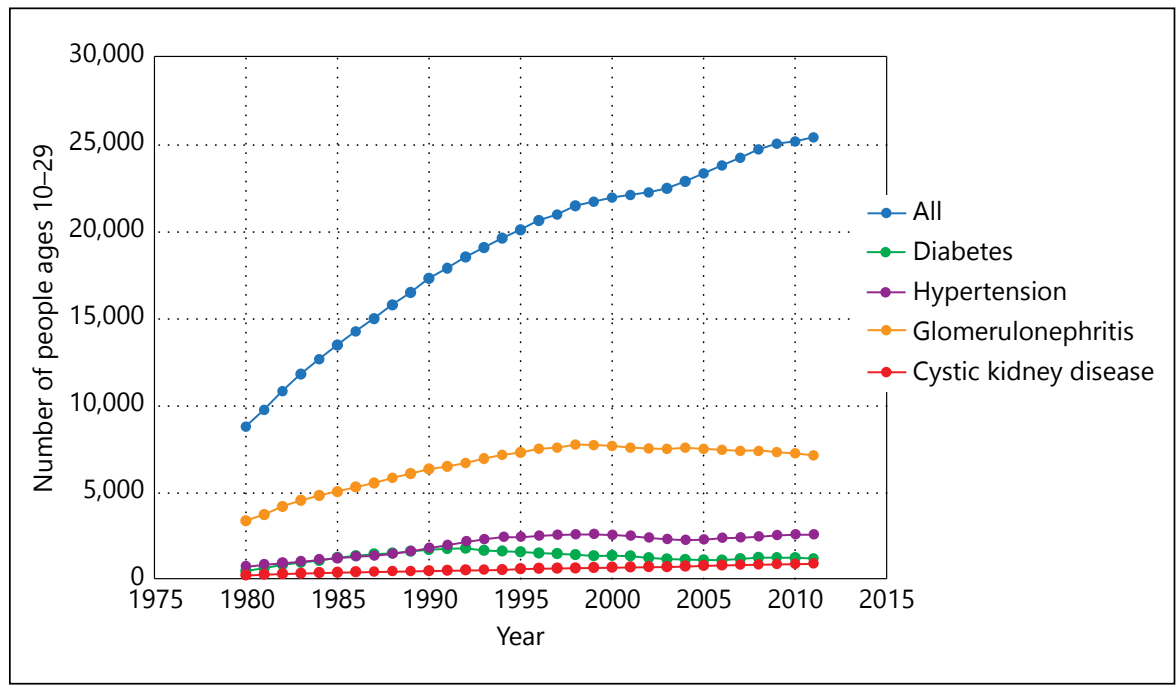

script is to characterize this unique population and enable providers to assist AYA with self-management and transition preparation to achieve better health and health-related quality of life outcomes.

\section{The Adolescent/Young Adult with Chronic Kidney Disease or End-Stage Kidney Disease}

Since 1980, the number of patients aged 10-29 with end-stage kidney disease (ESKD) in the United States has tripled to over 25,000 individuals $(76 \%$ between the ages of 20 and 29 years) as depicted in figure 1. Patients with adolescent-onset ESKD have a 10-year survival of greater than $85 \%$, but their survival remains lower than agematched peers [1]. The etiology of ESKD varies by age. Kidney disease diagnosed at a younger age is most commonly due to congenital anomalies of the kidneys and urinary tract, whereas in the older age it is most often due to glomerular disease [4]. In fact, the USRDS reports glomerular disease is the most prevalent diagnosis among AYA and diabetes is the least common [5].

AYA with CKD/ESKD have challenges associated with their age and diagnosis [6], including: (a) normally maturing brain that will not reach its full developmental state until the patient reaches his or her mid-to-late 20s (and possibly later for those with cognitive impairment [7-9]); (b) depression, lower socialization skills and self-esteem that impair their ability to achieve the developmental milestones of those without a chronic condition $[7,8]$; (c) higher risk behaviors and nonadherence to treatment[3]; (d) transitioning out of the parental home and loss of parental supervision; (e) developing a personal identity and peer recognition; (f) overcoming physical appearance such as dysmorphic features and/or short stature; (g) performing school or work-related tasks when cognition is impaired, and (h) learning to self-manage a condition with numerous medications, procedures, and nutritional demands [10].

Compared to other age groups with CKD/ESKD AYA are at risk for complications related to poor health selfmanagement and nonadherence $[3,11,12]$. Among patients through age 55 who received a first renal transplant, those between 14 and 16 years of age had the highest risk of graft loss [13]. Outcomes were worse starting at one year, and amplified at 3,5, and 10 years post-transplant and Black adolescents were at disproportionately high risk of graft loss [13]. Given the rate of complications, the International Societies of Nephrology and Pediatric Nephrology developed a consensus statement recommending transition and self-management-related activities for both pediatric and adult-focused nephrology practices [14].

\section{Health Self-Management and the Process of HCT}

Self-management is defined in the Oxford dictionary as 'management of or by oneself; the taking of responsibility for one's own behavior and well-being'. For patients with CKD/ESKD, self-management requires following a prescribed diet, tracking blood pressure, and other tasks to produce the best patient outcomes [15]. In-center hemodialysis patients tend to have worse self-management compared to those who receive home therapies [16]. 
Health self-management was associated with dialysis treatment adherence in a Southeastern US sample of 119 hemodialysis and 51 peritoneal dialysis adults [12]. Smoking also correlated significantly with nonadherence, negative perceived effects of ESKD, and shortened treatments [12].

Self-advocacy is the patients' willingness to make decisions, negotiate with providers, control their own treatment and act positively in their own self-interest (e.g., getting second opinions or partnering with providers to identify the treatments that work best for the patients). It is an important part of managing a chronic illness and translates into improved adherence [11]. Self-management of health-related aspects, in addition to everyday aspects of life affected by CKD/ESKD, is crucial to achieve positive health outcomes [17].

In CKD/ESKD, self-management encompasses four areas: nutrition, blood pressure, laboratory results, and medication management [18]. Self-management interventions must include all of these domains and be tailored to each individual's circumstances. Self-management programs for kidney disease are being developed $[18,19]$ and self-management measurement tools for CKD/ESKD are being validated $[11,19]$. Clinicians need to gauge their patients' self-management behaviors and provide these interventions when needed. Preparing patients to selfmanage CKD/ESKD and its comorbidities requires interdisciplinary collaboration [20].

Health care transition (HCT) preparation is defined by the Society of Adolescent Medicine as 'the purposeful, planned movement of adolescents and young adults with chronic physical and medical conditions from child-centered care to the adult-oriented health-care system' [21]. For AYA with CKD/ESKD, self-management preparation (in the adult-focused settings) and transition preparation (starting in the pediatric setting, but continuing in the adult-focused setting) should occur at clinic visits with every member of the health team [19]. AYA with CKD/ESKD, their family, and their multi-disciplinary team of providers in both the pediatric and adult-focused practices must collaborate to make the HCT process successful. During this process, AYA need to have the opportunity to develop the skills to successfully self-manage their health conditions [22]. Annual self-management and/or HCT readiness assessments are administered to guide interventions based on skill mastery. Tools to measure [19] and assist with this HCT process are available at our website: www.med.unc.edu/transition.

The Health Care Transition Research Consortium (HCTRC), an international and interdisciplinary collabo-

Youth with CKD/ESKD and

Self-Management ration, was created to develop effective HCT programs and best practices by sharing experiences and publications in monthly calls and semi-annual meetings (https:// sites.google.com/site/healthcaretransition/). This consortium developed a model for HCT preparation that includes four domains: individual-level factors, family/social support, the environment, and the health-care system [22]. While a successful HCT remains loosely defined, providers encouraging AYA to take on more responsibility for their care and guiding the HCT preparation are essential for successful outcomes [23]. It is recommended that HCT and self-management preparation should start between the ages of 12 and 14 and continue into the adultfocused health setting [24]. Generic, customizable, and $\mathrm{CKD} / \mathrm{ESKD}$-specific transition planning and toolkits to help providers are available to facilitate the preparation process $[19,25]$. CKD/ESKD patients who do not receive HCT preparation are at risk for poor health outcomes including quality of life, access to medical care, disease outcomes, future education, and employment $[26,27]$.

Both barriers and facilitators exist for AYA as they develop self-management skills. Barriers to effective selfmanagement and transition preparation include: denial, depression, poor coping, low literacy, low cognition, poor patient education, prolonged dependence on parents or significant others, a complex medical regimen with a high burden of care and lack of integrated health systems or electronic health records. Facilitators to self-management and HCT preparation include the implementation of a formal program preparing AYA to self-manage their condition, parents willing to relinquish the management of treatment, and having access to an electronic health record with a patient portal. For all providers, it is critical to identify strategies engaging AYA to become successful self-managers of their disease, enlist the help of behavioral cognitive therapy, and engage the support of patients' peers or significant others.

\section{Self-Management/Transition Preparation Activities for CKD/ESKD}

A planned HCT preparation allows the youth to achieve better outcomes at adult-focused health settings [28]. This includes diagnosing and measuring HCT readiness to guide tailored clinical interventions. There are some generic tools that measure HCT in youth with chronic health conditions that are being validated [19, 27, 29]; but they need to be correlated with health outcomes. 
Table 1. Suggestions to facilitate self-management and/or health care transition (HCT) for adolescents and young adults (AYA) in the pediatric- or adult-focused health care setting

Pediatric setting Adult setting

Ensure private and confidential discussions in the clinic setting starting at age 12

Consider the AYA's support system/relationships to decide who should be present during some portion of the clinic visit. Invite the AYA to bring three written questions at each visit

Ask the AYA to bring three written questions about their health at each clinic visit

Encourage AYA to attend therapeutic camps to show the AYA how other patients their age manage their disease

Encourage AYA to attend regular support groups Measure HCT readiness and/or self-management skills annually

Identify ecological and psychosocial factors that may hinder or facilitate HCT and/or self-management

Develop and organize an HCT team to communicate regularly with adult-focused teams
Encourage active involvement in psychosocial tasks and activities promoting normalization

Encourage attendance to regular support groups

Measure self-management skills annually

Identify ecological and psychosocial factors that may hinder or facilitate self-management

Develop and organize a dedicated HCT team to communicate regularly with pediatric teams
In order to adequately assist AYA in becoming effective health self-managers and best prepare them for HCT, it is important to develop programs and activities that take into consideration what we have already learned. For AYA with CKD/ESKD, effective understanding of their disease, treatment and self-management/transition preparation often requires extensive concept repetition. This is particularly true for those with any type of cognitive impairment. The preparation process should focus on both the patient and their family. Careful consideration of health self-management/transition preparedness should be given to patients with severe neurodevelopmental disabilities, tailoring the process to suit both the patient and their caregiver equally, given that the caregiver plays a major role in transition planning in these cases (i.e., planning and obtaining guardianship) [30].

Understanding the ecological and psychosocial factors that predict health outcomes in CKD/ESKD provides insight for designing effective interventions [31]. Relationships with family members, access to social or emotional support, and conflicting situations of life fluctuate during the transition years. Changes occur during the adolescence and emerging adulthood, such as leaving high school and childhood friends, moving away from home, job seeking and going away to college. Social or romantic relationships may be especially emotional and powerful. During this period, some individuals experience a sense of instability. For AYA with CKD/ESKD, the need to transfer to adult-focused care may increase their vulnerability [26].

Our experiences with patients in the self-management and HCT field since 2006 inform the HCT and self-management program development process. The self-management and HCT transition program at UNC Chapel Hill has shown that facilitators for self-management and/ or HCT include: female gender, Caucasian race, private health insurance and a two-parent household $[26,31]$. This critical information allows health providers to tailor self-management and transition services such that no AYA is disadvantaged within the system due to societal or environmental complexities. As HCT programs are integrated into health-care systems, tracking patient-reported outcomes and utilizing patient portals with electronic health records will uncover additional information.

\section{How Can We Actively Facilitate the Self-Management and HCT Process?}

Research and experience have aided health-care providers in understanding the factors involved in HCT preparation. Active facilitation of the self-management and HCT process is the next step. There are multiple ways to facilitate successful self-management and HCT (see table 1). One way to accomplish these goals is to complete an annual (or bi-annual) assessment of transition readi- 
ness beginning at ages $12-14$, and provide services accordingly. Annual transition assessments provide the clinician, associated staff, family members, and the AYA with important information regarding preparedness for a complex health-related transition, as well as insight into self-management and self-advocacy skills. Information obtained from transition readiness assessments such as the TRxANSITION Scale [19] is expansive and includes illness knowledge, prescription knowledge, adherence behaviors, nutrition/dietary knowledge related to their condition, self-management skills, knowledge regarding reproduction issues, knowledge of importance of future plans for a job or school, knowledge of insurance, knowledge of how they would go about finding a new healthcare provider, and the identification of ongoing support. Once a baseline of self-management skills and transition readiness is obtained, services and interventions can be customized to address the gaps in the AYA's knowledge. This in turn should help increase health outcomes and health-related quality of life.

While the bulk of tasks necessary to facilitate HCT should be completed on the pediatric side before transfer, self-management preparation must continue in the adultfocused setting. Having a clearly defined HCT team and identifying one or more transition coordinators who are in charge of overseeing the process can help facilitate selfmanagement and HCT. The most effective teams are interdisciplinary and incorporate tools to assess and support the decisions made by the patient. There should also be a written self-management or HCT plan for each patient and his or her family. Support for the parents to facilitate their child in their self-management needs to take place, with timely advice about health and drug insurance planning. Using a checklist of critical tasks and milestones is also a key component. An up-to-date medical passport (a wallet-sized card including diagnoses, medication dosing/purpose and allergies) like the one used at our program, empowers AYA to be responsible self-managers. This passport and other transition resources are available at http://www.med.unc.edu/transition.

In the adult-focused setting, physicians will need to review the developmental stages of adolescents and the effect of chronic disease on the timing of these stages [32]. Resources at the adult site, at minimum, should include a designated transition coordinator or leader. This leader could be a trained professional (nurse, social worker, patient care manager/coordinator) with an interest in this area. Ideally, it is good for a dedicated urologist and reproductive specialist (with proficiency in congenital genitourinary malformations) to be a part of the team. The clinic should have a young adult-friendly area for peer support and information exchange, with a mechanism to facilitate both pre and post-transfer conversations between the pediatric- and adult-focused teams. To optimize patient outcomes, communication between providers and the AYA and their family is paramount, but it must be in a manner that suits the developmental, literacy, and cultural backgrounds of the patients. Other strategies to facilitate AYA transition and/or self-management capabilities are listed here:

1) Measure self-management or transition readiness to provide guided patient education and interventions;

2) Start patient transition/self-management preparation at age 12, continuing through the adult-focused settings;

3) Involve AYA in decision-making;

4) Invite AYA to bring three written questions about their health at each visit;

5) Encourage AYA to see health providers without their parents/significant others for at least a portion of the visit, assuring confidentiality;

6) Provide AYA with the most simple medical/diet regimen possible;

7) Provide adaptive behavioral strategies as memory aids and repeat concepts;

8) Facilitate referrals to CKD/ESKD support groups;

9) Refer to therapeutic camps allowing the AYA to learn skills and relate with and share experiences with peers undergoing similar experiences;

10) Acknowledge their strengths/resilience and optimize coping ability.

\section{Conclusions}

The number of adolescents/young adults with endstage kidney disease continues to increase, and this age group is vulnerable to poor health outcomes. It is important to examine the self-management and transition from pediatric through adult health care to improve outcomes in these patients. There has been progress in these areas through the development of self-management programs, HCT readiness assessment tools, and identification of socioecologic risk factors. We know that self-advocacy, selfmonitoring, and medication adherence are crucial factors of self-management for AYA with ESKD, and have found that HCT preparation can focus on these areas. We have also found that peer support from therapeutic camps may be helpful in this preparation.

There are still deficiencies and barriers to self-management and HCT for AYA with ESKD. We are yet to reach 
a consensus regarding the outcomes of successful HCT preparation, and the tools that assess self-management/ HCT readiness are being validated. Not all clinics have multidisciplinary transition coordination teams to prepare AYA for adult care. Similarly, adult providers may not have AYA-friendly clinics, making it difficult to ad- dress the social and environmental factors influencing self-management and HCT readiness.

It is imperative for pediatric- and adult-focused providers to make efforts to improve the self-management and HCT of AYA with ESKD, and ultimately help these patients achieve better health-related quality of life and outcomes.

\section{References}

$\nabla_{1}$ Ferris ME, Gipson DS, Kimmel PL, Eggers $>_{12}$ Kutner NG, Zhang R, McClellan WM, Cole SA: PW: Trends in treatment and outcomes of survival of adolescents initiating ESRD care in the United States. Pediatr Nephrol 2006;21: 1020-1026.

- Andreoni KA, Forbes R, Andreoni RM, Phillips G, Stewart H, Ferris M: Age-related kidney transplant outcomes: health disparities amplified in adolescence. JAMA Intern Med 2013;173:1524-1532.

- 3 Koshy SM, Hebert D, Lam K, Stukel TA, Guttmann A: Renal allograft loss during transition to adult healthcare services among pediatric renal transplant patients. Transplantation 2009;87:1733-1736.

4 USRDS: http://www.usrds.org/reference.aspx (last visited August 20, 2014).

5 Usvyat LA, Haviv YS, Etter M, Kooman J, Marcelli D, Marelli C, et al: The MONitoring Dialysis Outcomes (MONDO) initiative. Blood Purif 2013;35:37-48.

-6 Ferris ME, Mahan JD: Pediatric chronic kidney disease and the process of health care transition. Semin Nephrol 2009;29:435-444.

7 Gipson DS, Hooper SR, Duquette PJ, Wetherington CE, Stellwagen KK, Jenkins TL, et al: Memory and executive functions in pediatric chronic kidney disease. Child Neuropsychol 2006; 12:391-405.

8 Groothoff JW, Grootenhuis MA, Offringa M, Stronks K, Hutten GJ, Heymans HS: Social consequences in adult life of end-stage renal disease in childhood. J Pediatr 2005;146:512-517.

9 Bartosh SM, Leverson G, Robillard D, Sollinger HW: Long-term outcomes in pediatric renal transplant recipients who survive into adulthood. Transplantation 2003;76:1195-1200.

-10 So TY, Layton JB, Bozik K, Farrington E, Gipson PE, Gibson K, Primack W, et al: Cognitive pharmacy services at a pediatric nephrology and hypertension clinic. Ren Fail 2011;33:1925.

11 Curtin RB, Walters BA, Schatell D, Pennell P, Wise M, Klicko K: Self-efficacy and self-management behaviors in patients with chronic kidney disease. Adv Chronic Kidney Dis 2008; 15:191-205.

\section{Psychosocial predictors of non-compliance in} haemodialysis and peritoneal dialysis patients. Nephrol Dial Transplant 2002;17:93-99.

13 Andreoni A, Forbes R, Andreoni RM, Phillips G, Stewart H, Ferris M: Age-related kidney transplant outcomes: health disparities amplified in adolescence. JAMA Intern Med 2013;173:1524-1532.

14 Watson AR, Harden P, Ferris M, Kerr PG, Mahan J, Ramzy MF: Transition from pediatric to adult renal services: a consensus statement by the International Society of Nephrology (ISN) and the International Pediatric Nephrology Association (IPNA). Pediatr Nephrol 2011;26:1753-1757.

-15 Brennan P, Safran C: Report of conference track 3: patient empowerment. Int J Med Inform 2003;69:301-304.

16 Curtin RB, Sitter DC, Schatell D, Chewning BA: Self-management, knowledge, and functioning and well-being of patients on hemodialysis. Nephrol Nurs J 2004;31:378-386.

17 Curtin RB, Mapes D, Schatell D, BurrowsHudson S: Self-management in patients with end stage renal disease: exploring domains and dimensions. Nephrol Nurs J 2005;32:389-395.

18 Ong SW, Jassal SV, Porter E, Logan AG, Miller JA: Using an electronic self-management tool to support patients with chronic kidney disease (CKD): a CKD clinic self-care model. Semin Dial 2013;26:195-202.

19 Ferris ME, et al: A clinical tool to measure the components of health-care transition from pediatric care to adult care: the UNC TR(x) ANSITION scale. Ren Fail 2012;34:744-753.

20 Sevick MA, Trauth JM, Ling BS, Anderson RT, Piatt GA, Kilbourne AM, et al: Patients with Complex Chronic Diseases: perspectives on supporting self-management. J Gen Intern Med 2007;22:438-444.

21 Blum RW, et al: Transition from child-centered to adult health-care systems for adolescents with chronic conditions. A position paper of the Society for Adolescent Medicine. J Adolesc Health 1993;14:570-576.

22 Betz CL, et al: The health care transition research consortium health care transition model: a framework for research and practice. J Pediatr Rehabil Med 2014;7:3-15.
23 U.S. Department of Health and Human Services, Health Resources and Services Administration, Maternal and Child Health Bureau: The National Survey of Children with Special Health Care Needs Chartbook 2009-2010. Rockville, Maryland, U.S. Department of Health and Human Services, 2013.

24 Cooley WC, Sagerman PJ; American Academy of Pediatrics; American Academy of Family Physicians; American College of Physicians; Transitions Clinical Report Authoring Group: Supporting the health care transition from adolescence to adulthood in the medical home. Pediatrics 2011;128:182-200.

25 The National Health Care Transition Centers 'Got Transition': http://www.gottransition. org/ (accessed August 29).

26 Bell LE, Ferris ME, Fenton N, Hooper SR Health care transition for adolescents with CKD-the journey from pediatric to adult care. Adv Chronic Kidney Dis 2011;18:384-390.

27 Sawicki GS, Lukens-Bull K, Yin X, Demars N, Huang I: Measuring the transition readiness of youth with special healthcare needs: validation of the TRAQ - Transition Readiness Assessment Questionnaire. J Pediatr Psychol 2011;36:160-171.

28 Callahan ST, Winitzer RF, Keenan P: Transition from pediatric to adult-oriented health care: a challenge for patients with chronic disease. Curr Opin Pediatr 2001;13:310-316.

29 Fredericks EM, Dore-Stites D, Well A, Magee JC, Freed GL, Shieck V, James Lopez M: Assessment of transition readiness skills and adherence in pediatric liver transplant recipients. Pediatr Transplant 2010;14;944-953.

30 Cheak-Zamora NC, et al: Disparities in transition planning for youth with autism spectrum disorder. Pediatrics 2013;131:447-454.

31 Javalkar K, Fenton N, Cohen S, Ferris M: Socioecologic factors as predictors of readiness for self-management and transition, medication adherence, and health care utilization among adolescents and young adults with chronic kidney disease. Prev Chronic Dis 2014;11:E117.

Ferris/Cuttance/Javalkar/Cohen/Phillips/ Bickford/Gibson/Ferris/True 